Case Report

\title{
A Cluster of Three Cases of Hantavirus Pulmonary Syndrome among Canadian Military Personnel
}

\author{
Leighanne O. Parkes, ${ }^{1}$ Trong Tien Nguyen, ${ }^{1}$ Jean Longtin, ${ }^{2,3}$ Marie-Claude Beaudoin, ${ }^{4}$ \\ Julie Bestman-Smith, ${ }^{4}$ Donald C. Vinh, ${ }^{1}$ Guy Boivin, ${ }^{2}$ and Vivian G. Loo ${ }^{1}$ \\ ${ }^{1}$ Division of Infectious Diseases and Department of Medical Microbiology, McGill University Health Centre, \\ McGill University, 1001 Boulevard Décarie, Montréal, QC, Canada H4A 3J1 \\ ${ }^{2}$ Infectious Diseases Research Center of the CHU de Québec, Université Laval, 2705 Boulevard Laurier, Québec, QC, Canada G1V 4 G2 \\ ${ }^{3}$ Laboratoire de Santé Publique du Québec, 20045 Chemin Ste-Marie, Sainte-Anne-de-Bellevue, QC, Canada H9X 3R5 \\ ${ }^{4} \mathrm{CHU}$ de Québec-Université Laval, Hôpital de l'Enfant Jésus, 1401 18e rue, Québec, QC, Canada G1J $1 Z 4$
}

Correspondence should be addressed to Vivian G. Loo; vivian.loo@mcgill.ca

Received 2 December 2015; Accepted 14 March 2016

Academic Editor: Marianna Ofner

Copyright (C) 2016 Leighanne O. Parkes et al. This is an open access article distributed under the Creative Commons Attribution License, which permits unrestricted use, distribution, and reproduction in any medium, provided the original work is properly cited.

Hantavirus pulmonary syndrome (HPS) is a rare illness in eastern Canada. We present three cases of HPS among military personnel in Quebec. The three cases shared a common exposure to mouse excreta while engaged in military training in Alberta, a western province of Canada.

\section{Introduction}

The Hantavirus genus (family Bunyaviridae) contains more than 20 species, each typically associated with a single rodent reservoir, resulting in geographically distinct human diseases. The clinical spectrum of Hantavirus infection includes nephropathia epidemica, hemorrhagic fever with renal syndrome, and Hantavirus pulmonary syndrome (HPS) [1]. HPS presents with a viral prodrome followed by fulminant noncardiogenic pulmonary edema.

HPS was first described in 1993 in South-Western USA. Canada's first case was recognized in 1994. As of December 31, 2014, 109 Canadian cases of HPS have been laboratoryconfirmed, averaging 5 cases yearly, mainly in the western provinces of Canada (Figure 1) [2]. The Sin Nombre strain is most commonly associated with HPS in Canada and the United States, reflecting the ubiquitous distribution of its reservoir, the deer mouse, Peromyscus maniculatus.

Until recently, only a single laboratory-confirmed case of Hantavirus infection has been reported in 2004 from eastern Canada, in the province of Quebec [3]. In this report, we describe an unusual cluster of three new cases of HPS diagnosed in Quebec in June and July of 2015.

\section{Patient 1}

In late June 2015, a healthy 22-year-old male presented to a community hospital in Montreal, Quebec, with vomiting, abdominal pain, and diarrhea for two days. He had returned from four weeks of military training in Alberta from April to May 2015. He was febrile at $39.3^{\circ} \mathrm{C}$ and tachycardic but his exam was otherwise unremarkable. Broad spectrum antibiotics were initiated for possible sepsis. Laboratory investigations are shown in Table 1.

The following day, the patient rapidly deteriorated, requiring intubation for hypoxic respiratory failure. Chest radiograph revealed bilateral pulmonary infiltrates with effusions (Figure 2). Due to refractory hypoxia and hypoperfusion, he was transferred to a tertiary care center ICU for extracorporeal membrane oxygenation (ECMO). Blood, urine, and stool cultures were negative. Respiratory virus PCR from nasopharyngeal swab was also negative for the respiratory pathogens. The patient was extubated following gradual improvement and he left hospital without sequelae.

Based on his epidemiological risk factors, Hantavirus serology was performed and was positive for both Hantavirus Sin Nombre IgG and IgM. This prompted public 
TABLE 1: Results of laboratory investigations on admission.

\begin{tabular}{lccc}
\hline Lab values & Patient 1 & Patient 2 & Patient 3 \\
\hline WBC $\left(* 10^{9} \mathrm{~g} / \mathrm{L}\right)$ & $53.41(4.50-11.00)$ & $7.1(4.8-10.8)$ & $16.75(4.20-10.00)$ \\
Neutrophil $(\mathrm{abs})\left(* 10^{9} \mathrm{~g} / \mathrm{L}\right)$ & $30.72(1.80-7.70)$ & $2.98(2.00-6.50)$ & $9.23(1.90-7.00)$ \\
Lymphocyte $(\mathrm{abs})\left(* 10^{9} \mathrm{~g} / \mathrm{L}\right)$ & $13.58(1.00-4.80)$ & $1.70(1.20-4.00)$ & $2.86(1.00-3.50)$ \\
Monocyte $(\mathrm{abs})\left(* 10^{9} \mathrm{~g} / \mathrm{L}\right)$ & $7.85(0.00-0.80)$ & $0.43(0.10-0.90)$ & $2.10(0.20-0.95)$ \\
Platelets $\left(* 10^{9} \mathrm{~g} / \mathrm{L}\right)$ & $35(140-450)$ & $61(150-400)$ & $41(150-360)$ \\
Hemoglobin $(\mathrm{g} / \mathrm{L})$ & $203(135-175)$ & $174(140-180)$ & $200(135-170)$ \\
Hematocrit $(\mathrm{L} / \mathrm{L})$ & $0.640(0.420-0.520)$ & $98(0.420-0.520)$ & $116(55-105)$ \\
Creatinine $(\mathrm{umol} / \mathrm{L})$ & $121(55-110)$ & $24 \%(0-8)$ & $7 \%(0-8)$ \\
Band cells & $26 \%(0-8)$ & \\
Additional & Plasmacytoid lymphocytes, smudge cells, and blasts & Plasmacytoid lymphocytes & Plasmacytoid lymphocytes \\
\hline
\end{tabular}

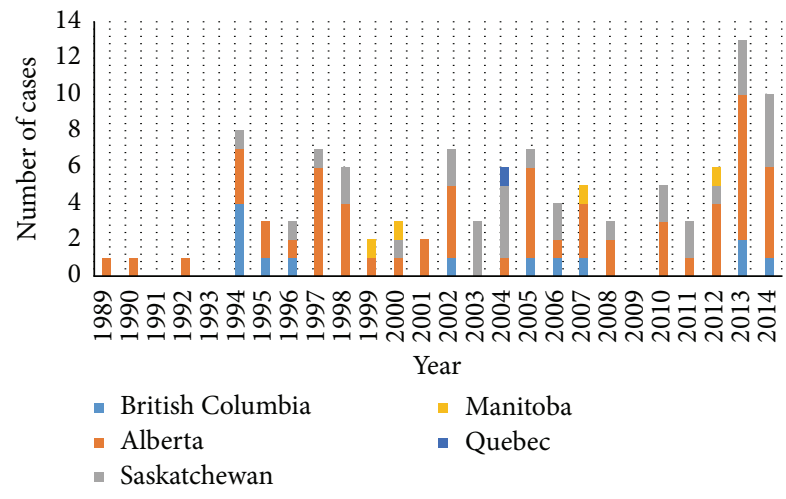

FIGURE 1: Number of Hantavirus cases in Canada from 1989 to 2014. Reproduced and adapted with permission from Drebot et al.

health notification. PCR targeting the conserved region of Hantavirus $\mathrm{M}$ segment detected the presence of Hantavirus from a nasopharyngeal swab sample that was procured on day six of illness. Further Hantavirus typing was performed by direct sequencing of the amplicon with sequence analysis using NCBI Basic Local Alignment Search Tool (BLAST) demonstrating similarity to Sin Nombre reference strains from Montana and Alberta.

\section{Patient 2}

In late June 2015, a 32-year-old male known for asthma and smoking presented to a Quebec City hospital. He had participated in the same military training as patient 1 , during the same time period. Two days prior to admission, he developed nonproductive cough, fever, shortness of breath, myalgias, and headache. The patient was initially afebrile but tachycardic and hypotensive (BP $85 / 47 \mathrm{mmHg}$ ). Chest radiograph revealed multifocal interstitial opacities. Levofloxacin was given for possible atypical pneumonia. Laboratory investigations are shown in Table 1.

The patient became more hypoxic over 48 hours and was intubated. His antimicrobial regimen was expanded. Bronchoalveolar lavage (BAL) was negative for bacterial and fungal cultures, Pneumocystis, Mycoplasma, and Chlamydophila
PCR, as was urinary legionella antigen. The patient gradually improved and was extubated without further sequelae.

Prompted by the public health alert, serology was performed, confirming positive Hantavirus IgG and IgM. Hantavirus PCR performed on the BAL specimen was negative.

\section{Patient 3}

In early July 2015, a healthy 30-year-old male presented to another Quebec City hospital with malaise, fever, cough, and dyspnea for three days. He developed vomiting prior to presenting. He had participated in the same military training at the same time as the previous two patients. He was febrile at $40.8^{\circ} \mathrm{C}$ and hypoxic with an oxygen saturation of $93 \%$ on room air. Chest radiograph revealed bilateral interstitial opacities with effusions. He received broad spectrum antibiotics for presumed complicated pneumonia. Laboratory investigations are shown in Table 1. The patient required noninvasive positive pressure ventilation (NIPPV) for hypoxia.

Cultures from BAL revealed normal flora and were negative for Pneumocystis, Legionella, mycobacteria, or fungi. Blood cultures and Histoplasma urinary antigen were negative. This patient did not require intubation and rapidly improved over several days.

Hantavirus serology was IgG positive at 1/400, but IgM equivocal. Repeat serology revealed a rise in IgG titre to $1 / 1600$. Hantavirus PCR performed on the BAL specimen was negative.

\section{Epidemiology}

The three patients were enrolled in the Canadian Armed Forces and based at Valcartier, Quebec. There was no history of foreign deployment. They had returned from a largescale military training exercise in Alberta that ran from April 20 until May 23, 2015, and involved 6,750 military personnel from Canada, the United States, and the United Kingdom. Patients reported mouse sightings and rodent excreta in the campsite and near their tents. They were exposed to aerosolized soil through activities such as military vehicle driving, trench excavation, live fire field exercises, and detonation of ammunition shells. Although the patients were 


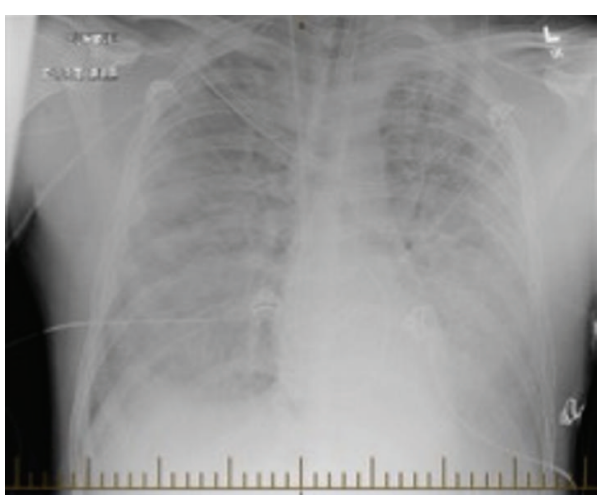

(a)

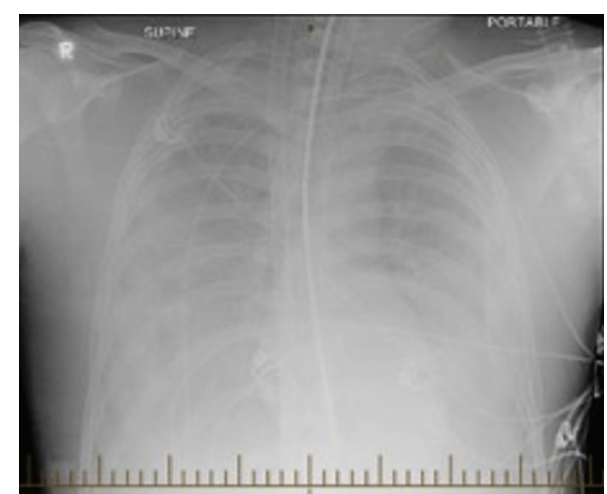

(b)

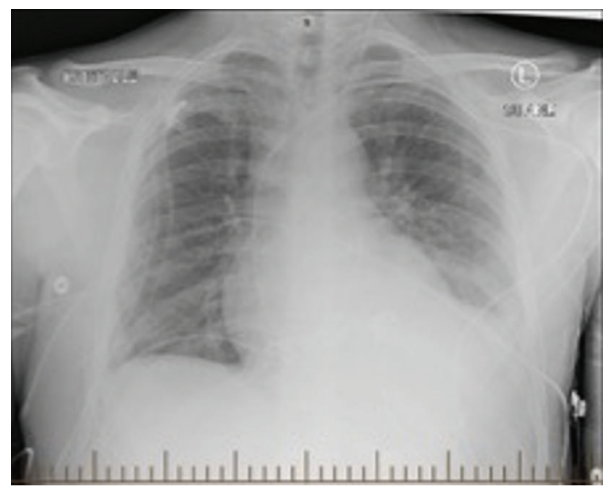

(c)

FIGURE 2: Radiographic progression of pulmonary disease in patient 1. (a) Chest radiograph on presentation; (b) chest radiograph on day two of admission following ECMO; (c) chest radiograph on day 10 of admission following extubation.

from different battalions, they participated in a common field exercise during their final week of training. The last case of Hantavirus from this region was reported in 1999 (M. Maher, pers. comm.).

\section{Discussion}

HPS is a rare respiratory syndrome in Canada, often associated with considerable morbidity and mortality, with a case fatality rate of $29 \%$ [2]. Human Hantavirus infection results from inhalation of aerosolized viral particles and subsequent infection of endothelial cells, resulting in capillary leakage, which in turn leads to the clinical features and laboratory anomalies seen in HPS [4-6].

Following an incubation period of up to 33 days, HPS classically begin with a 3-5-day phase of fever with nonspecific symptoms including headache, nausea, anorexia, diarrhea, abdominal pain, and malaise [1]. Typical laboratory abnormalities are sensitive but lack specificity: hemoconcentration, thrombocytopenia, granulocytosis with left shift, absence of myeloid toxic changes, and increased immunoblasts on smear [7]. A cardiopulmonary phase follows, marked by progressive respiratory symptoms and diffuse, bilateral radiographic pulmonary infiltrates. Hypoxia, hemodynamic instability, and distributive shock can progress over 24 to 48 hours and account for most case fatalities [8-10].
All three patients, following an incubation of 4-5 weeks, exhibited a similar febrile prodrome and typical laboratory abnormalities and progressed to hypoxic respiratory failure requiring cardiorespiratory support (ECMO, mechanical ventilation, or NIPPV).

The association between military activity and Hantavirus is not new. The first reported outbreak of "Korean hemorrhagic fever" occurred during the 1950 Korean War. Similar outbreaks were reported among US troops in Germany [11] and Korea [12], Croatian Army soldiers [13], and NATO forces in Bosnia and Herzegovina [14]. Contrary to this, autochthonous infection in the Canadian military service is uncommon. Reported cases in Canada and the United States typically involve patients from rural locations, engaged in peridomestic cleaning and agriculture or exposed in rodentinfested dwellings $[2,10,15]$. The patients in our cluster do not fit this usual profile. To our knowledge this represents the first cluster of cases among military personnel in Canada.

\section{Conclusion}

We report a rare occurrence of HPS identified in a cluster of three patients in Quebec, who shared a common epidemiological exposure. This case series outlines the importance of the consideration of HPS as a part of the differential diagnosis for severe respiratory illness. It also highlights outdoor 
military activity in areas of high Hantavirus prevalence in the rodent population, as a risk factor for HPS. Furthermore, we emphasize the importance of public health notification and its role in identifying and tracking new cases in order to mitigate further infection risk.

\section{Disclosure}

Work originated from Division of Infectious Diseases and Department of Medical Microbiology, McGill University Health Centre, Montréal, QC, Canada.

\section{Competing Interests}

The authors declare that they have no competing interests relevant to this paper.

\section{Authors' Contributions}

Drs. Nguyen and Parkes contributed equally to this paper.

\section{References}

[1] D. C. Vinh and J. M. Embil, "Hantavirus pulmonary syndrome: a concise clinical review," Southern Medical Journal, vol. 102, no. 6, pp. 620-625, 2009.

[2] M. A. Drebot, S. Jones, A. Grolla et al., "Hantavirus pulmonary syndrome in Canada: an overview of clinical features, diagnostics, epidemiology and prevention," Canada Communicable Disease Report, vol. 41, no. 6, pp. 65-69, 2015, http:// www.phac-aspc.gc.ca/publicat/ccdr-rmtc/15vol41/dr-rm41-06/ ar-02-eng.php.

[3] E. Weir, "Hantavirus: 'tis the season," CMAJ, vol. 173, no. 2, p. $147,2005$.

[4] G. Schönrich, D. H. Krüger, and M. J. Raftery, "Hantavirusinduced disruption of the endothelial barrier: neutrophils are on the payroll," Frontiers in Microbiology, vol. 6, pp. 1-9, 2015.

[5] J. Hepojoki, A. Vaheri, and T. Strandin, "The fundamental role of endothelial cells in hantavirus pathogenesis," Frontiers in Microbiology, vol. 5, pp. 1-7, 2014.

[6] B. E. Steinberg, N. M. Goldenberg, and W. L. Lee, "Do viral infections mimic bacterial sepsis? The role of microvascular permeability: a review of mechanisms and methods," Antiviral Research, vol. 93, no. 1, pp. 2-15, 2012.

[7] L. Dvorscak and D. R. Czuchlewski, "Successful triage of suspected hantavirus cardiopulmonary syndrome by peripheral blood smear review-a decade of experience in an endemic region," American Journal of Clinical Pathology, vol. 142, no. 2, pp. 196-201, 2014.

[8] C. B. Jonsson, L. T. M. Figueiredo, and O. Vapalahti, "A global perspective on hantavirus ecology, epidemiology, and disease," Clinical Microbiology Reviews, vol. 23, no. 2, pp. 412-441, 2010.

[9] D. C. Watson, M. Sargianou, A. Papa, P. Chra, I. Starakis, and G. Panos, "Epidemiology of Hantavirus infections in humans: a comprehensive, global overview," Critical Reviews in Microbiology, vol. 40, no. 3, pp. 261-272, 2014.

[10] R. Verity, E. Prasad, K. Grimsrud et al., "Hantavirus pulmonary syndrome in Northern Alberta, Canada: clinical and laboratory findings for 19 cases," Clinical Infectious Diseases, vol. 31, no. 4, pp. 942-946, 2000.
[11] J. Clement, P. Underwood, D. Ward, J. Pilaski, and J. LeDuc, "Hantavirus outbreak during military manoeuvres in Germany," The Lancet, vol. 347, no. 8997, p. 336, 1996.

[12] E. Pon, K. T. McKee Jr., B. M. Diniega, B. Merrell, A. Corwin, and T. G. Ksiazek, "Outbreak of hemorrhagic fever with renal syndrome among U.S. Marines in Korea," American Journal of Tropical Medicine and Hygiene, vol. 42, no. 6, pp. 612-619, 1990.

[13] R. Mulić and D. Ropac, "Epidemiologic characteristics and military implications of hemorrhagic fever with renal syndrome in Croatia," Croatian Medical Journal, vol. 43, no. 5, pp. 581-586, 2002.

[14] A. Markotic, J. W. LeDuc, D. Hlaca et al., "Hantaviruses are a likely threat to NATO forces in Bosnia and Herzegovina and Croatia," Nature Medicine, vol. 2, no. 3, pp. 269-270, 1996.

[15] B. Knust and P. E. Rollin, "Twenty-year summary of surveillance for human hantavirus infections, United States," Emerging Infectious Diseases, vol. 19, no. 12, pp. 1934-1937, 2013. 


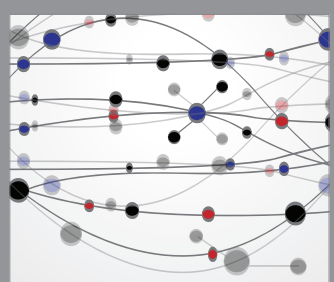

The Scientific World Journal
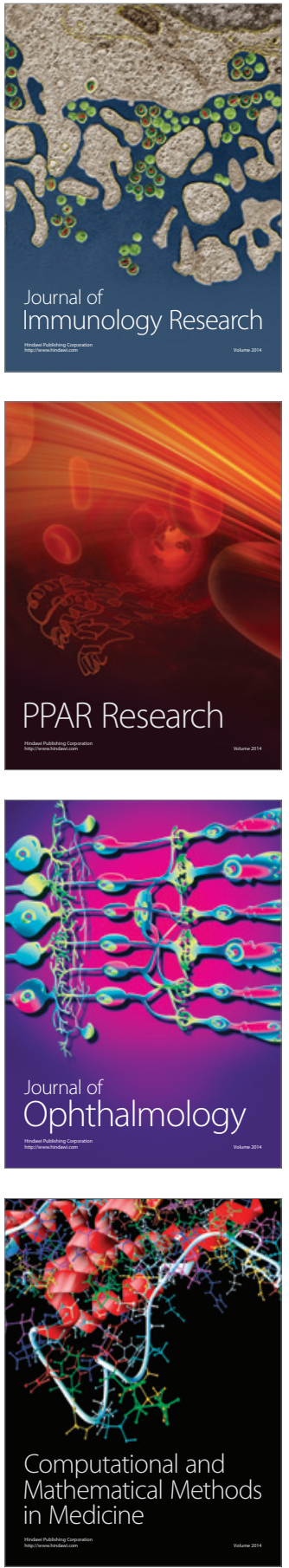

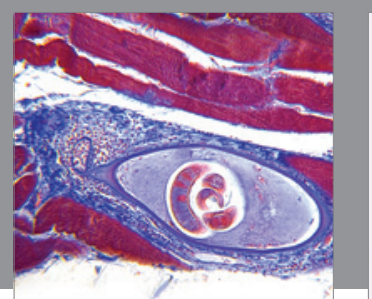

Gastroenterology Research and Practice

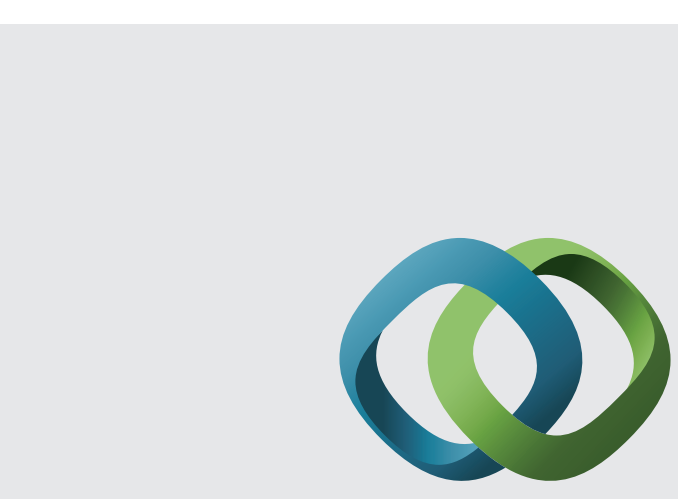

\section{Hindawi}

Submit your manuscripts at

http://www.hindawi.com

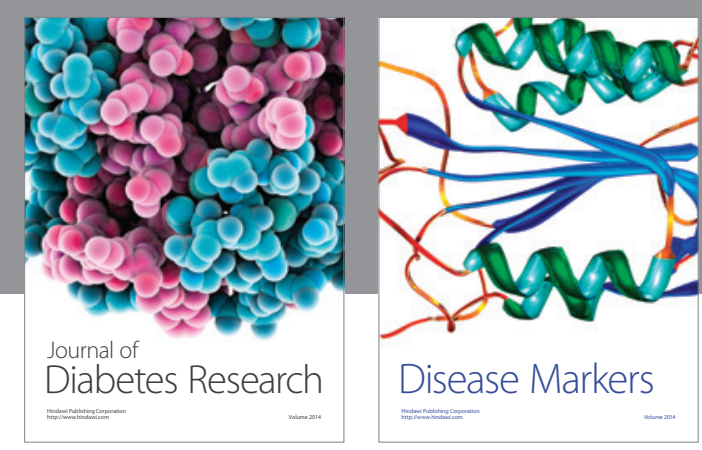

Disease Markers
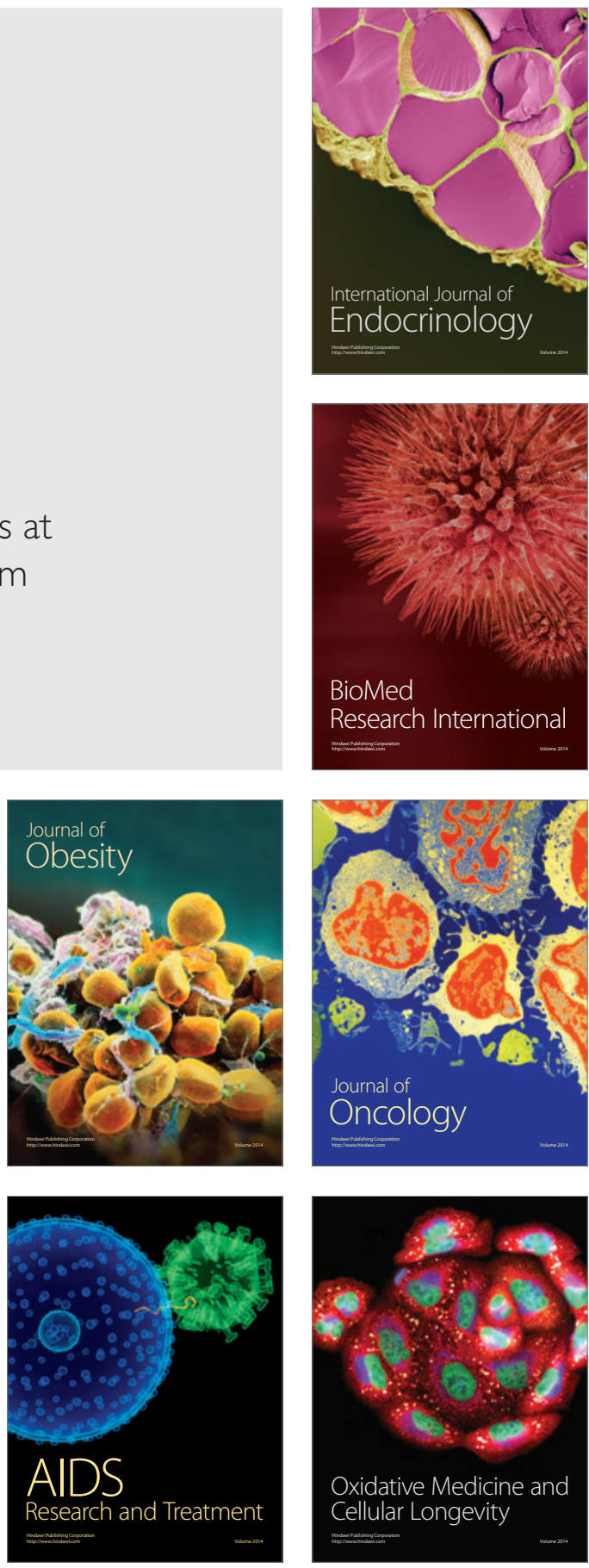